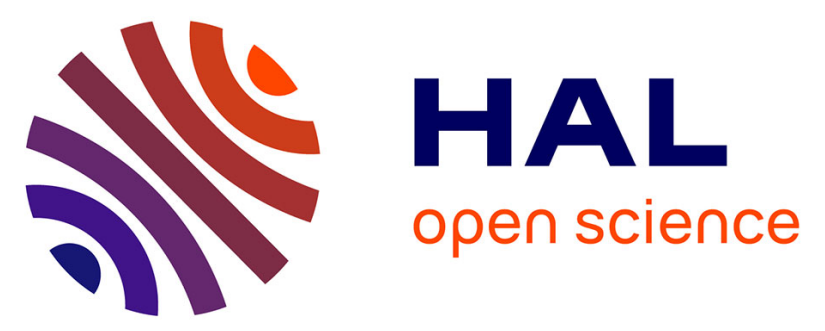

\title{
Design and Implementation of TD-LTE-Based Real-Time Monitoring System for Greenhouse Environment Temperature
}

\author{
Xin Zhao, Yang Jiao, Lianjun Yu, Chuanhong Zhang
}

\section{- To cite this version:}

Xin Zhao, Yang Jiao, Lianjun Yu, Chuanhong Zhang. Design and Implementation of TD-LTE-Based Real-Time Monitoring System for Greenhouse Environment Temperature. 9th International Conference on Computer and Computing Technologies in Agriculture (CCTA), Sep 2015, Beijing, China. pp.170-177, 10.1007/978-3-319-48354-2_18. hal-01614217

\author{
HAL Id: hal-01614217 \\ https://hal.inria.fr/hal-01614217
}

Submitted on 10 Oct 2017

HAL is a multi-disciplinary open access archive for the deposit and dissemination of scientific research documents, whether they are published or not. The documents may come from teaching and research institutions in France or abroad, or from public or private research centers.
L'archive ouverte pluridisciplinaire HAL, est destinée au dépôt et à la diffusion de documents scientifiques de niveau recherche, publiés ou non, émanant des établissements d'enseignement et de recherche français ou étrangers, des laboratoires publics ou privés.

\section{(c)(1)}

Distributed under a Creative Commons Attribution| 4.0 International License 


\title{
Design and Implementation of TD-LTE-based Real-time Monitoring System for Greenhouse Environment Temperature
}

\author{
Xin Zhao ${ }^{1,2, \mathrm{a}}$, Yang Jiao ${ }^{1,2, \mathrm{~b}}$, Lianjun $\mathrm{Yu}^{3, \mathrm{c}}$, Chuanhong Zhang ${ }^{4, \mathrm{~d}, *}$ \\ ${ }^{1}$ College of Biological and Agricultural Engineering, Jilin University,Changchun 130022,China; \\ ${ }^{2}$ Sixth Middle School in Changchun,Changchun 130000,China; \\ ${ }^{3}$ Changchun City Academy of Agricultural Sciences, Changchun 130111, China \\ ${ }^{4}$ Changchun University of Science and Technology, Changchun 130600, China \\ ajlndzx@sina.com, ${ }^{\mathrm{b}} 61516131 @$ qq.com, ${ }^{\mathrm{c}} 120142901 @ \mathrm{qq} . c o m,{ }^{\mathrm{d}} 15844005115 @ 163 . c o m$,
}

\begin{abstract}
With the characteristics of fast transferring speed and wide coverage of TD-LTE network, the research designs and implements the TD-LTE-based Real-time Monitoring System for Greenhouse Environment Temperature. The TD-LTE network, which is connected with the data server of the Internet, can transmit the real-time temperature data that sensor collects to the data server. The $\mathrm{B} / \mathrm{S}$ mode-based data server can realize data query, analysis and other functions. This monitoring system solves the problems that previous monitoring systems are slow and can not be transmitted at a distance, and it can also meet the need of the real-time data acquisition that precision agriculture needs.
\end{abstract}

Keywords: TD-LTE, Precision agriculture, Data collection, long-distance monitoring

\section{Introduction}

Compared with the commonly used wired network, wireless network is a new type of network formation. It is no longer dependent on the network cables which the traditional wired network depends on, eliminating the need for network cabling, effectively saving the cost, being used more and more conveniently. In the past, most wireless networks construct network with the help of WIFI, ZigBee, Bluetooth and so on. This method can implement the wireless transmission, but the transmission distance is near. If you want to transfer to a far distance, you need get access to the Internet. TD-LTE fully Time Division Long Term Evolution, also simply LTE-TDD, is one of the duplex technologies used in mobile communication technology. It is also one of the international standards led by China. TD-LTE can directly get access to the Internet, which makes up for the shortcomings of the traditional wireless network and greatly improves the efficiency of the wireless network. ${ }^{[1-4]}$

The greenhouse, also called greenhouse, can keep heat. In the season that is not suitable for plant growth, it can ensure the normal growth of plants, and ensure the output. The greenhouse is an important part of modern agriculture. ${ }^{[5]}$ In the modern 
precision agriculture, the control of the growth environment of crops in the greenhouse has been changed from the traditional personal management mode to the modern, centralized management mode. Highly centralized management can effectively reduce the management cost, can make timely and accurate judgment in the shortest time, ensure the normal operation of the greenhouse, and ensure the normal production of greenhouse crops. ${ }^{[6-9]}$ Greenhouse temperature is the basis of greenhouse planting and the coverage of centralized management is large, and the wireless network, which the traditional greenhouse temperature collection system used, is ZigBee mode, while ZigBee itself does not have the ability to get access to Internet, so the traditional greenhouse temperature monitoring system can only work in a small range, which is a problem for centralized management. ${ }^{[10-12]}$ Based on TDLTE network and embedded system, the greenhouse environment temperature monitoring system, which is comprehensive covering network, provides the wireless transmission mode that is not limited by the distance, and the efficient and fast data transmission service..

\section{Overall Design System}

The system consists of a wireless remote temperature acquisition system and an online data server based on WEB. The core of the greenhouse environment temperature acquisition system is embedded system, which uses the temperature sensor to collect the real-time data of the greenhouse environment temperature, and transmits the collected data to the data server on Internet through TD-LTE network. Database server is based on WEB technology, which achieve real-time monitoring, analysis and management of long-distance data. The overall structure of the system is shown in figure 1:

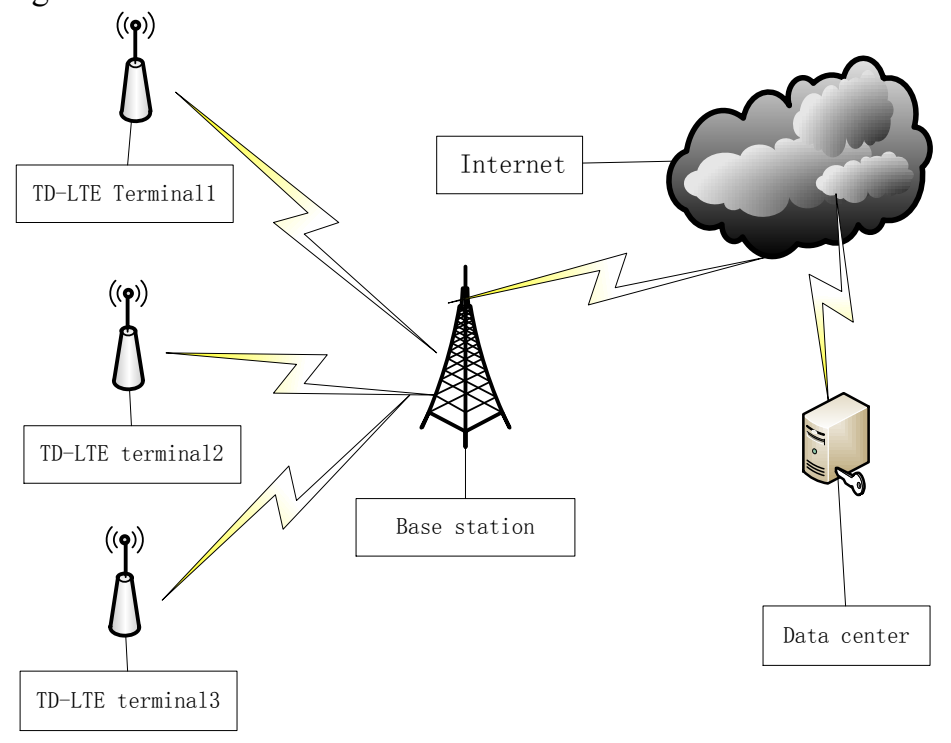

Fig. 1. Conventional and spectra after pretreatment 


\section{Temperature Acquisition Module Design}

\subsection{TD-LTE}

LTE, which is based on the OFDMA technology, is the global standard by the 3GPP organization. TD-LTE, also LTE-TDD, fully Time Division Long Term Evolution, is one of the duplex technologies used in mobile communication technology. It is developed by the world's major companies and operators covered by the 3GPP organization. It is also one of the international standards led by China. It is a followup evolution technology scheme of TD-SCDMA, and its network transmission speed is dozens of times as fast as TD-SCDMA. TD-LTE can be directly connected to the Internet, and functions with the Internet serve, which greatly improving the efficiency of wireless network.

HUAWEI B593S is the production of TD-LTE produced by HUAWEI. The device supports TD-LTE through the built-in DHCP function, and also down is compatible with TD-SCDMA and EDGE networks, which can achieve a comprehensive coverage of the network. In the network access mode, the device supports 802.11 and RJ45, and 802.11 supports the connection of multi-users, so the system mainly used RJ45 mode.

\subsection{Embedded}

The hardware of this system includes the embedded microprocessor and peripheral equipment. The system mainly consists of CPU chip, FLASH chip, SDRAM chip, DM9000 network card, sensor and other hardware components. And the CPU, which is the core of the whole hardware system, uses the ARM series embedded processor $\$ 3 \mathrm{C} 2440 \mathrm{~A}$ by Sanxing. SDRAM and FLASH chips are the system storage module. FLASH stores boot code, kernel, and file of system (including the applications), and SDRAM provides the running space for the system and applications and stores temporary data when system working.

\subsection{Operating System}

Linux is an operating system that can run on a variety of different hardware platforms. The software platform of the smallest embedded Linux system consists of three parts - the boot process, the kernel image and the file system. When the system is powered, firstly the guidance program is implemented, then the kernel is called and booted by it in order to realize the detection and drive of hardware system, finally mount the file system and run the applications.

Linux kernel is well-structured and integrated with the most parts of the mainstream of the hardware driver. With the help of the Linux kernel, the whole system is more stable. And Linux has the perfect network function, which can make our system get good network compatibility. The Linux core of the current standard is too large to the resource-constrained embedded system. So due to the features that embedded 
software and hardware can be cut, we need to abandon our unused modules in the kernel, leaving only the functions needed by the system.

\subsection{Data Communication}

In order to guarantee the fast, safe and efficient data communications between the acquisition system and the server, it is necessary to carry on a series of conventions for the communication heat preservation format between each other. The system uses the data preservation format as "verification code || node number|| date || temperature 1 || temperature 2 || temperature 3 \#\#", and the" || "is the data delimited identifier, and" \#\#" is the data end identifier. In the acquisition frequency of temperature data, the system can be accurate to 1 second, but, in fact, such a high accuracy is not necessary, so the system's acquisition time is set to 1 minute. Internet server intercepts properly the relevant data that the acquisition system sends and stores them in the database.

In order to guarantee the continuity and effectiveness of the data and prevent network outages and other emergencies, the system carries on the real-time transmission of data to the server, then backups them and stores the data to the embedded system. And at 0 o'clock everyday, the aggregated data will be sent to the server and be verified with server-side data so as to ensure the continuity and effectiveness of the data.

\section{Server}

The operating system of the server uses Linux operating system because Linux is the open source operating system. It has obvious advantages in terms of security. At the aspect of software cost, the windows system is charged and running software is also charged, so the cost is higher, while the Linux operating system is lower than the windows operating system and most running software are free software. At the aspect of centralized management, windows needs a large number of servers, while using Linux can effectively reduce the cost. Because Linux is selected as the operating system, Mysql is chosen to be the database. Mysql is also an open source database and it has the advantages, such as small size, fast speed and it can effectively guarantee the security of data storage.

\subsection{Data Reception}

Data transmission is through the Internet network, using the "standard" data communication format, so the server side intercepts the transmission data in terms of the calibrated data format. This program uses JAVA to write and the database uses Mysql to carry on the data storage. When the program is started, the process will automatically monitor the port to transmit data. Upon receipt of the data, the first data packet header data is verified to determine whether the data is sent to the client data, 
if verified on, then split the received data according to the agreed data format and stored them in the corresponding database.

\subsection{Data Monitoring}

The data monitoring platform features the real-time display for the greenhouse temperature data and analyzes the historical data. The monitoring platform is developed with the help of $\mathrm{B} / \mathrm{S}$ structure model. The advantage of this model is that the application core of the system is focused on the server, which simplifies the development, maintenance and usage of the system. In the client side, there does not need carry out a separate application development, the client can interact with the server information through the browser. The client is also not limited to the windows operating system, but both Linux and MAC can be used.

The server uses Linux as the operating system. Database uses MySQL as the database, uses JAVA to carry out the relevant WEB page development and uses apache as the middleware. The network construction can effectively ensure the stable running of the system and the interaction between the user and the database. Through the browser to get access to the WEB page, the client can monitor the real-time temperature of the remote greenhouse and can view, analyze and export the historical data.

\section{System Implementation}

\subsection{Data Acquisition}

When the embedded system is running, it will automatically take 1 minute to collect the temperature data in the greenhouse, as shown in Figure 2:

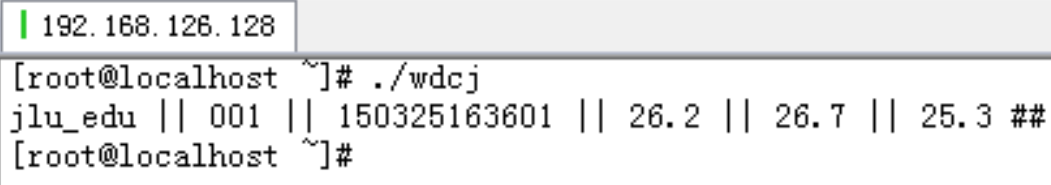

Fig. 2. Collect data

In addition to real-time transmission of data, the system also files historically data. At 0 o'clock daily, a full day's data will be uploaded to the server so as to ensure the continuity and effectiveness of data, as shown in Figure 3: 


\begin{tabular}{|c|c|c|c|c|c|}
\hline \multicolumn{6}{|c|}{ | 192.168.126.128 } \\
\hline \multicolumn{6}{|c|}{ [root@localhost "]\# cat h_time. log } \\
\hline jlu_edu & 001 & $1503251 \overline{6} 3401$ & 26.1 & 26.8 & 25.3 \# \\
\hline jlu_edu & 001 & 150325163501 & 26.1 & 26.8 & 25.2 \# \\
\hline jlu_edu & 001 & 150325163601 & 26.2 & 26.7 & 25.3 \# \\
\hline jlu_edu & 001 & 150325163701 & 26.2 & 26.7 & 25.2 \# \\
\hline jlu_edu & 001 & 150325163801 & 26.1 & 26.9 & 25.2 \# \\
\hline jlu_edu & 001 & 150325163901 & 26.2 & 26.9 & 25.1 \# \\
\hline jlu_edu & 001 & 150325164001 & 26.1 & 26.8 & 25.1 \# \\
\hline jlu_edu & 001 & 150325164101 & 26.1 & 26.8 & 25.1 \# \\
\hline jlu_edu & 001 & 150325164201 & 26.2 & 26.7 & 25.1 \# \\
\hline jlu_edu & 001 & 150325164301 & 26.2 & 26.7 & 25.2 \# \\
\hline jlu_edu & 001 & 150325164401 & 26.3 & 26.7 & 25.2 \# \\
\hline jlu_edu & 001 & 150325164501 & 26.3 & 26.7 & 25.2 \# \\
\hline jlu_edu & 001 & 150325164601 & 26.3 & 26.7 & 25.2 \# \\
\hline jlu_edu & 001 & 150325164701 & 26.2 & 26.8 & 25.2 \# \\
\hline jlu_edu & 001 & 150325164801 & 26.1 & 26.8 & $25.3 \#$ \\
\hline jlu_edu & 001 & 150325164901 & 26.1 & 26.9 & 25.2 \# \\
\hline jlu_edu & 001 & 150325165001 & 26.1 & 26.8 & 25.3 \# \\
\hline jlu_edu & 001 & 150325165101 & 26.1 & 26.9 & $25.4 \#$ \\
\hline
\end{tabular}

Fig. 3. Collect data

\subsection{Data monitoring}

In the data monitoring, the system not only displays the current temperature, but also display the recent 5 temperature state below so as to facilitate the observation of data trends, as shown in Figure 4: 


\begin{tabular}{|c|c|c|c|c|c|c|c|c|}
\hline \multirow[t]{13}{*}{$\square$ now data } & $x$ & + & & & & & & \\
\hline & & \multicolumn{7}{|c|}{ Now data } \\
\hline & From & n jlu_edu & $\checkmark$ & No 1 & $v$ & \multicolumn{3}{|c|}{ Search } \\
\hline & \multicolumn{8}{|l|}{ Now data } \\
\hline & & From & No & Date & Time & $\mathrm{T} 1$ & $\mathrm{~T} 2$ & T3 \\
\hline & 0 & jlu_edu & 1 & 150325 & 165301 & 26.1 & 27.0 & 25.4 \\
\hline & \multicolumn{8}{|c|}{ Recent data } \\
\hline & & From & No & Date & Time & $\mathrm{T} 1$ & $\mathrm{~T} 2$ & T3 \\
\hline & 1 & jlu_edu & 1 & 150325 & 165201 & 26.0 & 26.9 & 25.3 \\
\hline & 2 & jlu_edu & 1 & 150325 & 165101 & 26.0 & 26.9 & 25.4 \\
\hline & 3 & jlu_edu & 1 & 150325 & 165001 & 26.1 & 26.8 & 25.3 \\
\hline & 4 & jlu_edu & 1 & 150325 & 164901 & 26.1 & 26.9 & 25.2 \\
\hline & 5 & jlu_edu & 1 & 150325 & 164801 & 26.1 & 26.8 & 25.3 \\
\hline
\end{tabular}

Fig.4. Data monitoring

\subsection{Historical Data}

The system has a historical data filtering function and meanwhile supporting to export to excel, as shown in Figure 5: 
$\square$ Historical deta

Historical data

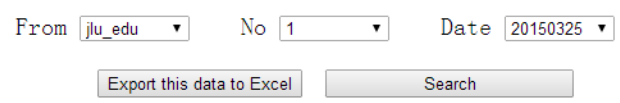

\begin{tabular}{|cccccccc|}
\hline & From & No & Date & Time & T1 & T2 & T3 \\
1 & jlu_edu & 1 & 150325 & 163401 & 26.1 & 26.8 & 25.3 \\
2 & jlu_edu & 1 & 150325 & 163501 & 26.1 & 26.8 & 25.2 \\
3 & jlu_edu & 1 & 150325 & 163601 & 26.2 & 26.7 & 25.3 \\
4 & jlu_edu & 1 & 150325 & 163701 & 26.2 & 26.7 & 25.2 \\
5 & jlu_edu & 1 & 150325 & 163801 & 26.1 & 26.9 & 25.2 \\
6 & jlu_edu & 1 & 150325 & 163901 & 26.2 & 26.9 & 25.1 \\
7 & jlu_edu & 1 & 150325 & 164001 & 26.1 & 26.8 & 25.1 \\
8 & jlu_edu & 1 & 150325 & 164101 & 26.1 & 26.8 & 25.1 \\
9 & jlu_edu & 1 & 150325 & 164201 & 26.2 & 26.7 & 25.1 \\
10 & jlu_edu & 1 & 150325 & 164301 & 26.2 & 26.7 & 25.2 \\
11 & jlu_edu & 1 & 150325 & 164401 & 26.3 & 26.7 & 25.2 \\
12 & jlu_edu & 1 & 150325 & 164501 & 26.3 & 26.7 & 25.2 \\
13 & jlu_edu & 1 & 150325 & 164601 & 26.3 & 26.7 & 25.2 \\
14 & jlu_edu & 1 & 150325 & 164701 & 26.2 & 26.8 & 25.2 \\
15 & jlu_edu & 1 & 150325 & 164801 & 26.1 & 26.8 & 25.3 \\
16 & jlu_edu & 1 & 150325 & 164901 & 26.1 & 26.9 & 25.2 \\
17 & jlu_edu & 1 & 150325 & 165001 & 26.1 & 26.8 & 25.3 \\
18 & jlu_edu & 1 & 150325 & 165101 & 26.0 & 26.9 & 25.4 \\
19 & jlu_edu & 1 & 150325 & 165201 & 26.0 & 26.9 & 25.3 \\
20 & jlu_edu & 1 & 150325 & 165301 & 26.1 & 27.0 & 25.4 \\
\hline & & & & & & Next &
\end{tabular}

Fig. 5. Historical Data

\section{Conclusions}

The system uses the embedded system and TD-LTE technology to implement the automatic collection of greenhouse environment temperature, and the collected data is transmitted to the data server side on Internet. Administrators can get access to the data server through the client's browser, and access contents information includes the current state of the greenhouse as well as browsing and analyzing the historical data. After testing, the system works stably and the transmission distance is more far, which provides a stable foundation for the modern agriculture centralized management. At the same time, the usage of the Linux operating platform has significantly reduced the cost of production, and produced certain economic benefits compared to the traditional windows platform. 


\section{Acknowledgment}

Funds for this research was provided by National 863 subjects (2012AA10A5064, 2013AA103005-04), Jilin province science and technology development projects(20110217), China Postdoctoral Science Foundation the 54th surface funded(2013M541308), Jilin University Young Teachers Innovation Project (450060491471).

\section{References}

1. GUAN Wei. Analysis on Key Technologies and Development Trend of LTE [J]. Guangxi Communication Technology, 2009(1):5-8.

2. Liao Jianjun. Discussion on TD-LTE:The Only TDD International Standard of LTE [J]. Value Engineering,2010(16):156.

3. Zhang Tongxu. An Overview of LTE and its Development [J]. Telecom Engineering Technics and Standardization, 2010(11):1-6.

4. HU Hai-ming, DONG Shao-jing, JIANG You-tian et al. Analysis of fourth generation mobile telecommunications technology[J]. 2011,32(5):1563-1567.

5. Ran Weigang, The Data Acquisition System for the Greenhouse [D]. Lanzhou University, 2010

6. LI Zhong-hua, WANG Guo-zhan, QI Fei. Current situation and thinking of development of protected agriculture in China [J].Chinese Agricultural Mechanization, 2012, (1): 07-10.

7. Yang Qichang, Wei Lingling, Liu Wenke et al.Current situation and development strategy of agricultural research in China [J]. China Agriculture Information, 2012(11):22-27

8. Hu Jian, Analysis on the Current Situation and Development of Modern Facilities Agriculture [J]. Journal of Agricultural Mechanization Research, 2012(07):245-248.

9. Ma.YT, Mathieu, Wubs. Parameter Estimation and Growth Variation Analysis in Six Capsicum Cultivars with the Functional-structural Model GreenLab[C]. Third International Symposium on Plant Growth Modeling, Simulation, Visualization and Applications. 2009:183-90.

10. LI Shi-hui, The research on the intelligent greenhouse monitoring system based on wireless sensor network [J].Journal of Jiangxi University of Science and Technology, 2013, 34 (1): 70-73.

11. Li Heqing, Lai Zhiyong, Zhang Xin. Intelligent Greenhouse Irrigation Subsystem Design and Implementation Based on ZigBeeTechnology [J].Journal of Agricultural Mechanization Research, 2014(1): 95-98+107.

12. Li Lei, Lin $\mathrm{T}$ usheng, Wu Zhenqiang. Development of a wireless safety monitoring system for laboratories based on Zigbee [J]. Experimental Technology and Management, 2011(1): 108-112. 